\title{
Deterministic Tourist Walks as an Image Analysis Methodology Based
}

\author{
André R. Backes ${ }^{1}$, Odemir M. Bruno ${ }^{1}$, Mônica G. Campiteli ${ }^{2}$, \\ and Alexandre S. Martinez ${ }^{2}$ \\ ${ }^{1}$ Instituto de Ciências Matemáticas e de Computação (ICMC) \\ Universidade de São Paulo (USP) \\ Avenida do Trabalhador São-carlense, 400 \\ 13560-970 São Carlos SP Brazil \\ backes@icmc.usp.br, bruno@icmc.usp.br \\ ${ }^{2}$ Faculdade de Filosofia, Ciências e Letras de Ribeirão Preto (FFCLRP) \\ Universidade de São Paulo (USP) \\ Avenida Bandeirantes, 3900 \\ 14040-901 Ribeirão Preto SP Brazil \\ monicacampiteli@pg.ffclrp.usp.br, asmartinez@ffclrp.usp.br
}

\begin{abstract}
Textures are important visual attribute used in image analysis. This paper presents a novel methodology, based on a deterministic walk, to texture analysis and texture characterization. Most of the methods adopted to classify textures deal with a defined fixed scale of texture. The method proposed here explores the set in all scales and is able to characterize efficiently different texture classes. The paper presents the deterministic walk technique and its results for two experiments using Brodatz images.
\end{abstract}

\section{Introduction}

Image Analysis is a field of computer vision and artificial intelligence responsible for the extraction of meaningful information from images. Among several attributes, texture is an important visual attribute used in image analysis. Texture analysis has a broad range of applications, such as: aid of diagnoses in medical images [1, remote sensing [2], analysis of geological images [3] and microscope images 4.

Although there is not a formal specification of the texture analysis, this attribute is directly related to the distribution of pixels in a certain region of the image. It represents an important source of information. Generally, textures can be classified as micro and macro textures according to the size of the set of pixels analyzed. By micro textures we mean the analysis of a small parts of the image, while for macro texture the analysis of the whole image. Most of the techniques used in image retrieval are devoted to the micro textures analysis [56]. The number of methods applied to macro textures is still restricted, due to the inherent difficulty in the analysis [7.

Recently we have proposed a new method for texture characterization based on a deterministic dynamics. Consider walkers leaving each pixel of an image. 
Each walker does not interact with the others. For a given time step, each walker has information about the eight next nearest neighbor pixels and moves towards the direction of minimum intensity difference among the ones that have not been visited in the previous $\mu$ time steps. For $\mu=0$, no dynamics is allowed. For $\mu=1$, the walker always goes to the mininum intensity difference direction, so that after a time transient, the trajectories end on cycles of period two. This cycle consists of two pixels having mutually the minimum intensity differences between themselves. More interesting cycle distributions occur for $\mu \geq 2$. In this case each trajectory, after a transient time, ends in a cycle with period $p \geq \mu=1$. In particular, cycles with period $p$ much greater that the memory $\mu$ are allowed. It is the range of cycle periods which allows the image analysis from the local scale of $\mu$ pixel up to a large scale of size of the number of pixels $N$.

Our main interest is to obtain an image signature from the time transient and cycle period joint distribution. A naive approach to this analysis has been described in Ref. 8, where we have shown the potential use of these joint distributions for texture analysis in images. A more sofisticated analysis, using the non-parametric Flexible Discriminant Analysis (FDA) on the joint distribution furnished a more reliable classification [9].

Our presentation is divided as follows. A brief review of the considered deterministic walk in Section 2. In Section 3, we modify these walks t apply them to image analysis. In Section 4, from the transient time and cycle period obtained from the walker trajectory we build texture signatures vectors. Also we set up experiment sets to compare the performance of two of the proposed texture signature vectors to 400 images of 40 different Brodatz's texture classes. In Section 5] we show the superior performance when multiple $\mu$ values are used. Finally in Section 6, the conclusions and improvement of the methods are discussed.

\section{Deterministic Tourist Walk}

Although not as thoroughly studied as random walks 1011, the study of deterministic walks has attained the interest of researchers 12131415 . Here, we are interested in exploring a partially self-avoiding deterministic walk algorithm, known as the tourist walk (TW) [161718 1920 21] for image analysis purposes.

The tourist walk algorithm can be pictorially viewed as a tourist wishing to visit $N$ cities randomly distributed in a map of $d$ dimensions. The tourist starts his route in a given city of this map and moves according to the following deterministic rule: go to the nearest city, which has not been visited in the last $\mu$ time steps. For $\mu \geq 1$, self-avoidance is limited to the memory window $\tau=$ $\mu-1$, which represents a characteristic time to the city to become attractive to the tourist again (refractory time). The trajectories can intersect outside this memory range. Each tourist trajectory consists of a transient part of length $t$ (new cities are visited) and a final cycle of period $p$ (no new cities are visited any longer). The trajectory is complex and depends strictly on the starting point and on the configuration of the data set. The only known relation that holds is $p \geq$ $\mu+1$. The tourist movements are entirely performed based on the neighborhood rank. These ranks are conveniently represented by a neighborhood table, which 
neglects the distances among the cities. This feature leads to invariance in scale transformations [21].

We call the attention to several aspects of these deterministic walks:

1. At each time interval the tourist moves from one city to another, regardless the distance between them.

2. Starting from different cities in the map, the tourist performs different trajectories with variable transient times (which can even be null $t=0$ ) and end in cycles with period $p \geq \mu+1$.

3. The trajectories are different for different initial conditions, but several trajectories can end in the same cycle with a given period $p$.

Although easy to formulate and not too complicated to implement numerically, this algorithm may present a complex behavior according to the chosen memory window $\mu$. This intriguing behavior can be captured with the transient time and period joint distribution $S_{d, \mu}^{(N)}(t, p)$. Here we show that the joint distributions $S_{d, \mu}^{(N)}(t, p)$ can be efficiently used as features for image analysis. In the following we show some examples of known analytical joint distributions for Poissonic landscape.

The deterministic tourist walk with memory $\mu=0$ is trivial since the walker does not move at each time step. The joint distribution is simply given by: $S_{d, 0}^{(N)}(t, p)=\delta_{t, 0} \delta_{p, 1}$, where $\delta_{i, j}$ is the Kronecker's delta.

With memory $\mu=1$, the walker must leave the visited city at each time step. The transient and period joint distribution is obtained for $N \gg 1$ [22]: $S_{d, 1}^{(\infty)}(t, p)=\Gamma\left(1+I_{d}^{-1}\right)\left(t+I_{d}^{-1}\right) \delta_{p, 2} / \Gamma\left(t+p+I_{d}^{-1}\right)$, where $t=0,1,2, \ldots, \Gamma(z)$ is the gamma function and $I_{d}=I_{1 / 4}[1 / 2,(d+1) / 2]$ is the normalized incomplete beta function. We stress that this transient time distribution has been calculated for Poissonic process. It does not lead to exploration of the random medium since after a short transient time, the tourist gets trapped in pairs of cities that are mutually nearest neighbors.

Interesting phenomena occur when greater memory values are considered. In this case, the cycle distribution is no longer peaked at $p_{\min }=\mu+1$, but presents a whole spectrum of cycles with period $p \geq p_{\min }$, with possible powerlaw decay [16 17 18]. Determinism imposes serious restrictions as it can be seen in $\mu=2$ one-dimensional systems, where all odd periods above $p_{\min }=3$ are forbidden, as well as the even period $p=6$.

\section{Modified Tourist Walk}

In the context of images $(d=2)$, one can consider each pixel as a point (or city). In the original algorithm the neighborhood table has size of the order $N^{2}$, where each point is ranked with respect to the remaining $N-1$ other points. Notice that $\mu$ ranges from zero to $N-1$, where all cities are visited (full self-avoiding circuit).

For images, the algorithm has been modified since each pixel interacts only with its first and second neighbors and the walker goes always to the direction 
of minimum intensity difference (gradient). Thus the neighborhood table has a maximum size of $N \times 8$. For open boundary condition, the surface and corner pixels have five and three next and second next nearest neighbors, respectively.

Starting from each pixel, the walker moves according to the deterministic rule of going to the pixel with nearest intensity compared to the present pixel intensity, so that the given intensity difference has not been attained in the preceeding $\mu$ steps. From these walks, the transient time and cycle period of the trajectories are calculated and the joint distribution is constructed.

The occurrence of ties is resolved by choosing the first pixel in the counter clockwise direction, preserving the deterministic nature of the algorithm. This approach is not invariant to image rotation. For a given image and the same image with $90^{\circ}$-tilt feeded to the algorithm, different joint distributions are produced. This suitable difference for texture characterization is due the deterministic way of resolving intensity difference ties.

Differently from the original problem, the tourist walk is not performed in a non-correlated random media but in a correlated medium, the image. Similarly to the previous studies [89], the image analysis consists exploring the joint distribution properties over the image and compose a texture signature curve.

\section{Experiments}

The tourist walk transient time and cycle period joint distributions have been obtained for different memory values using open boundary conditions to the images from the book of Brodatz 23. These images form a set largely used in computer vision and image processing literature as benchmark for texture analysis. In the computer experiment, each image has $200 \times 200$ pixels with 256 gray levels and 40 classes, with 10 samples each, have been employed. One example of each these 40 classes are dipicted in Figure 1. Experiments have been idealized to show the high potential of the method to analyze and characterize texture images.

\subsection{Transient Time and Cycle Period Joint Distribution}

It is important to stress that the transient time has the role of counting different intensity gradients while the cycle detects a kind of pattern that eventually an image may display. The memory $\mu$ has the role of setting the minimum pattern scale.

In Figure 2 three different classes of Brodatz's textures are dipicted and for each class, the transient time and cycle period joint distribution of the modified tourist walk is display for three different memory values. Observe the existence of a pattern for the joint distribution for each considered texture class. This stresses the potentiability to discriminate different texture classes from these distributions. Also, it is clear that the large the memory values are, the broader the distribution is. This means that different $\mu$ values sample different image characteristics indicating the possibility to have an optimum $\mu$ value for better 


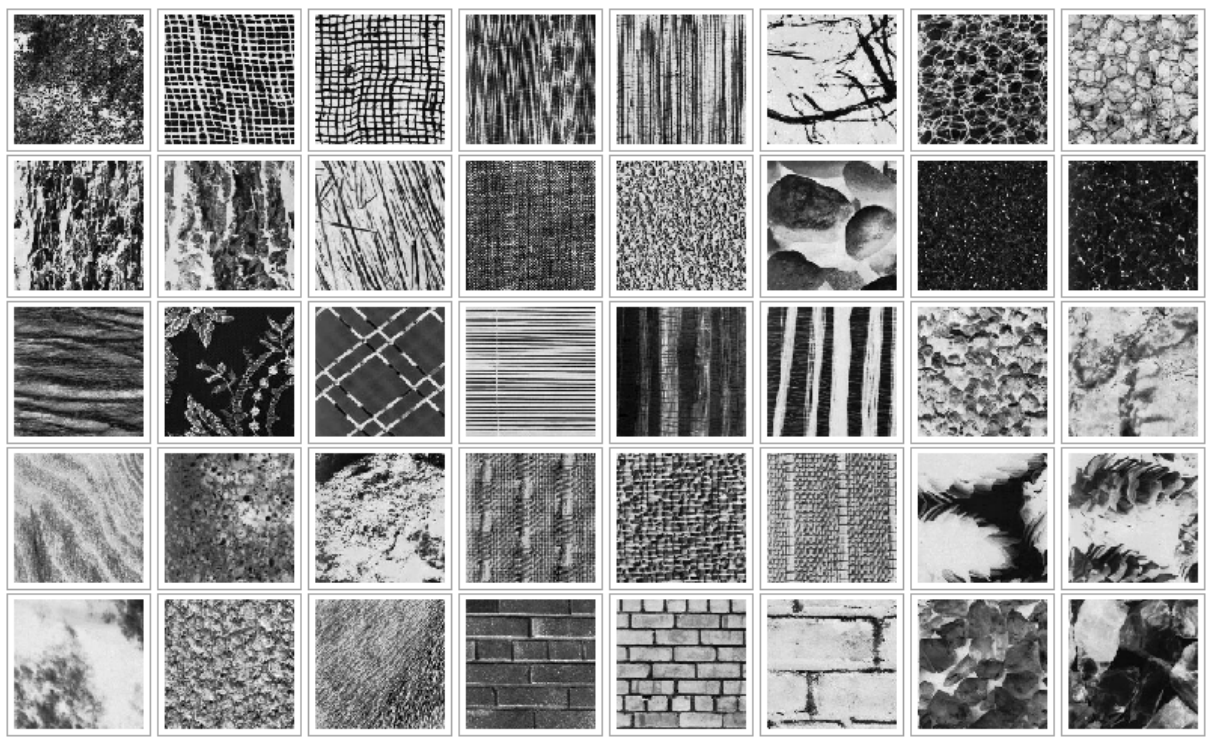

Fig. 1. One example of each of the 40 Brodatz's classes considered. Each image has $200 \times 200$ pixels and 256 grey levels.

image classification. The signature curves obtained from the joint distributions have been used to characterize and classify the images used in the experiment.

Operationally one has a three-dimensional array where one of the axis represents the transient time. The other axis represents the cycle period. When these two axes are combined, they represent the joint distribution $S_{\mu}^{(N)}(t, p)$. The third axis represents the memory, so that slices along the $\mu$ axis give $S_{\mu}^{(N)}(t, p)$.

A large amount of information is contained in this three-dimensional array. This implies to difficulties when dealing with pattern recognition. Our main objective is to extract texture information, in a simple form, using signatures.

\subsection{Texture Signature Vector}

Signatures concentrate the desired texture information to few elements of a vector. For instance, for images of $200 \times 200$ pixels, signatures are vectors is a typical size of 25 elements.

The signature curves are feature vectors extracted from the joint distributions and they are used to characterize and classify the images by the texture pattern. In the first approach the feature vector $\boldsymbol{\psi}$ is constructed from the joint distribution of a specific $\mu$ value. The parameters $t$ and $a$ indicate the maximum number of transient time and the maximum number of attractors to be considered, respectively:

$$
\boldsymbol{\psi}_{\mu}(t, a)=\left[S_{2, \mu}^{(N)}(0: t, \mu+1) S_{2, \mu}^{(N)}(0: t, \mu+2) \ldots S_{2, \mu}^{(N)}(0: t, \mu+a)\right] .
$$




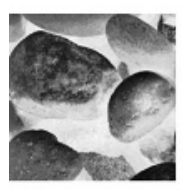

(a)

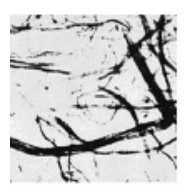

(e)

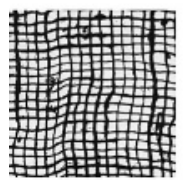

(i)

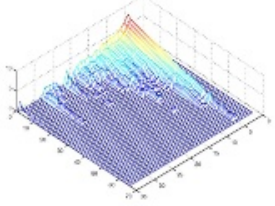

(b)

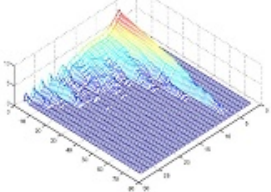

(f)

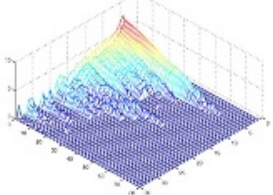

(j)

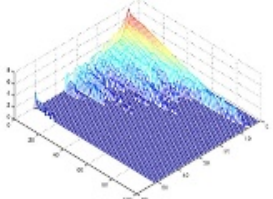

(c)

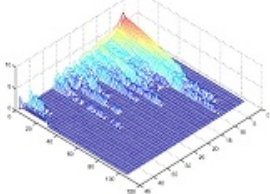

(g)

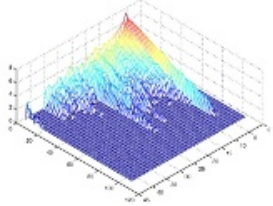

(k)

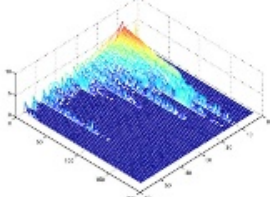

(d)

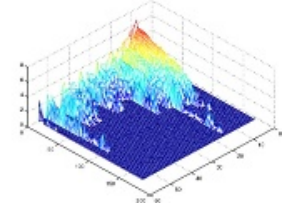

(h)

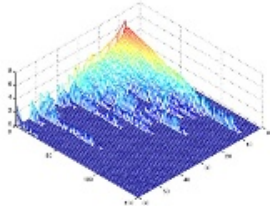

(1)

Fig. 2. Three different classes of Brodatz's textures are dipicted in panels from (a), (e) and (c). For each class, the transient time and cycle period joint distribution of the modified tourist walk is display for three different memory values: $\mu=2$ (pannels: (b), (f) and (j)), $\mu=3$ (pannels: (c), (g) and (k)) and $\mu=5$ (pannels: (d), (h) and (l)).

To study the influence of the memory on the time transient and cycle period distribution and consequently its influence in the sample classification, the experiments have been performed with $t=4$ and $a=5$ so that each signature vector has 25 elements. Experiments have been carried out for $\mu=2,3,5,7$ and 11.

In the second experiment only the first two time transients $(t=0$ and $t=1)$ and two cycle periods $a=2$ are considered for $\mu=2,3,5,7$ and 11 and the signature vector in this case is the concatenation of the simpler fixed signature vectors

$$
\boldsymbol{\varphi}\left(\mu_{i}, \mu_{f}\right)=\left[\boldsymbol{\psi}_{\mu_{i}}(2,2) \ldots \boldsymbol{\psi}_{\mu_{f}}(2,2)\right] \text {. }
$$

\subsection{Flexible Discriminant Analysis}

In both experiments, the Brodatz's images have been characterized by modified tourist walk texture signature. A discriminant data analysis technique has been performed, based on these features. The statistical analysis has been carried out with the R 2.1.1 system [24] and the employed technique has been the flexible discriminant analysis (FDA) 25. This is a generalization of linear discriminant analysis (LDA). It is more sofisticate analysis than LDA, once it uses 
non-parametric fits to achieve a more flexible classifier than LDA. The experimental results and the discussion about the performance of the modified tourist deterministic walks are presented in the following.

\section{Results}

Table 2 shows the confusion matrix obtained for the first experiment, i.e., when the $\mu$ values are not combined. The signature vector has the most 24 representative elements of the transient time and cycle period joint distribution of the modified tourist walk. This signature vector has been submitted to a flexible determinant analysis. Table 1 shows that as the memory $\mu$ increases, the error tends to increase.

The second set of experiments have been performed concatenating part of the $\mu$ image signature vector from the first set of experiments into a single image

Table 1. The error as a function of the memory $\mu$ for the fixed memory experiment

\begin{tabular}{rcccccc}
\hline$\mu$ & 2 & 3 & 5 & 7 & 11 & multiple $\mu$ \\
error & 0.1225 & 0.1775 & 0.2025 & 0.1725 & 0.2625 & 0.0525 \\
\hline
\end{tabular}

Table 2. Confusion matrix showing the classification results for 40 classes of texture images, for signature extracts for $\mu=5$. Classification error $=0.2025$.

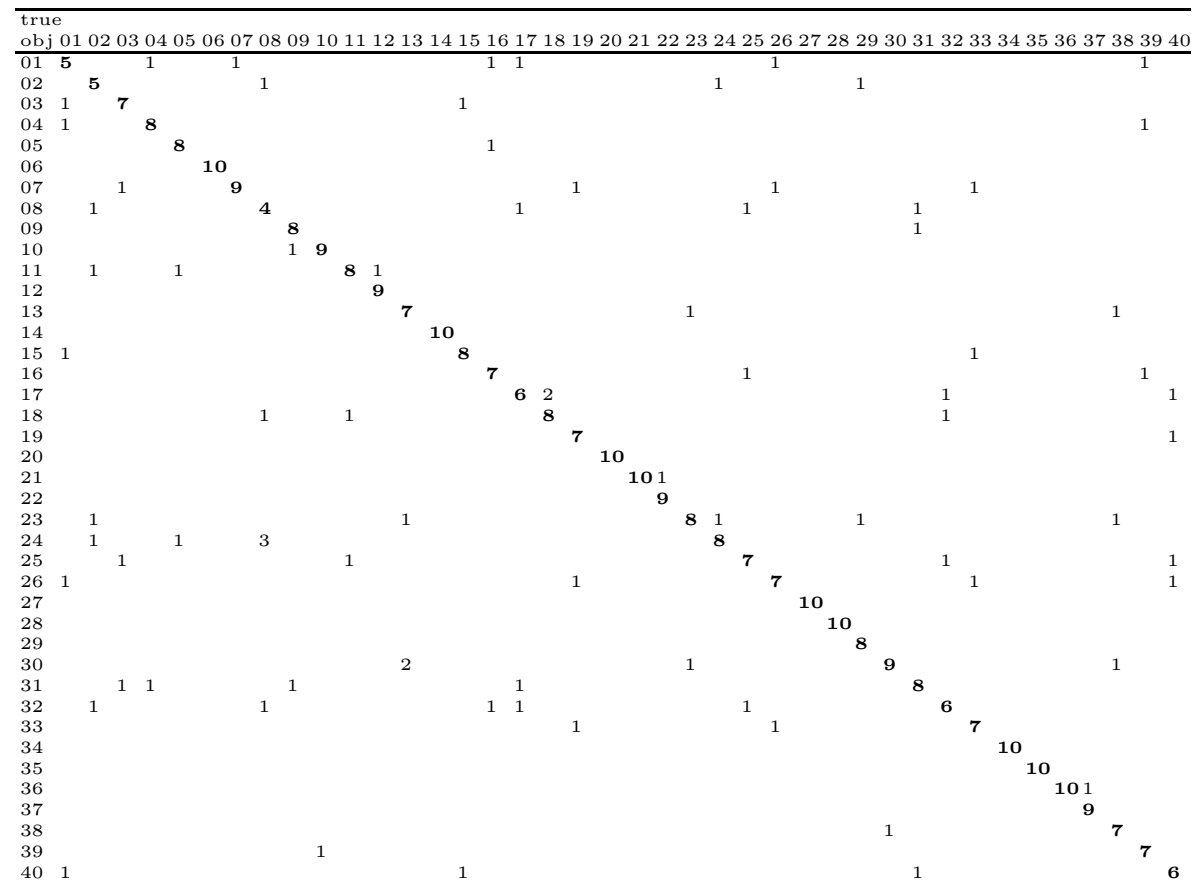


Table 3. Confusion matrix showing the classification results for 40 classes of texture images, for the combining $\mu$ method. Classification error $=0.0525$.

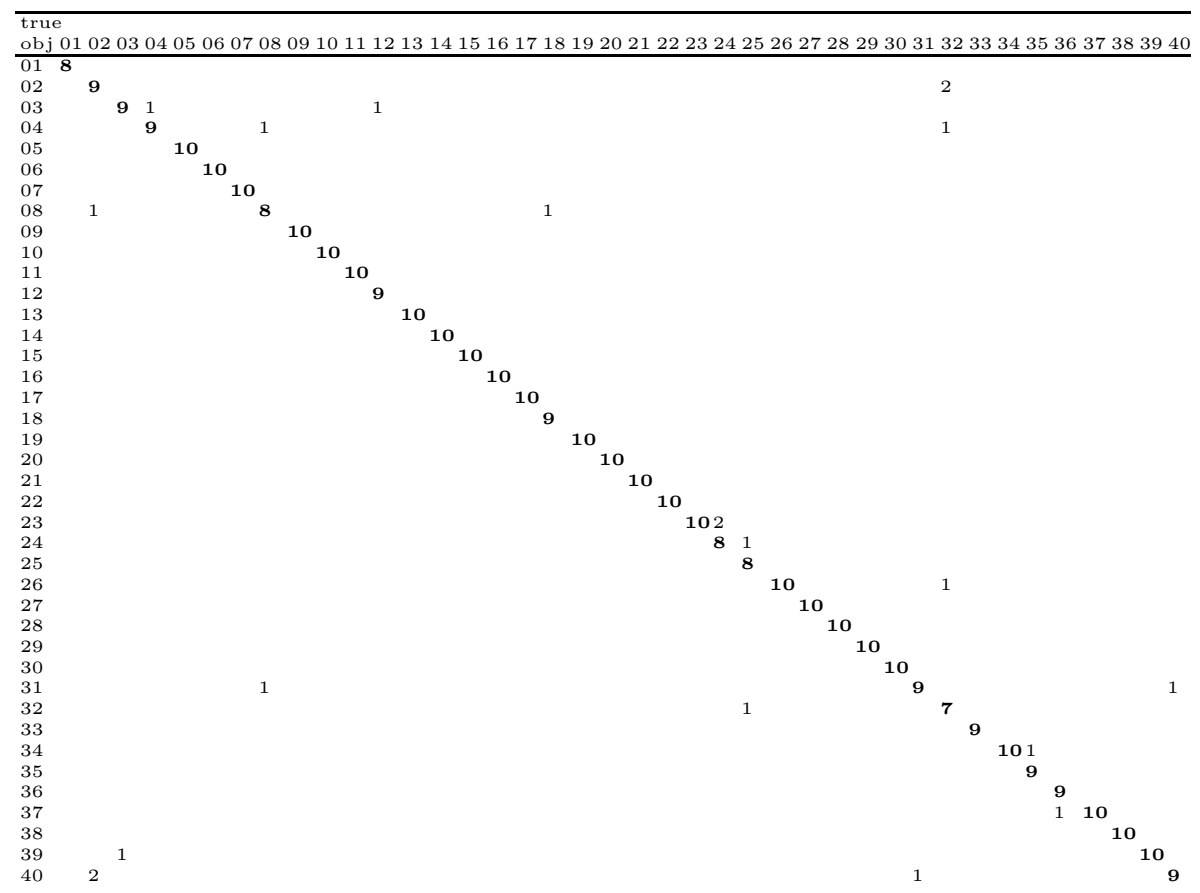

signature vector. This approach leads to a significant increase of the method classification capacity. The composed signature vector has been generated colecting the five more significant elements from each single $\mu$ signature vector leading to 25 elements. The confusion table of this set of experiments is presented in Table 3 and the error is of 0.0525 .

Collecting six elements from the single signature vector leading to 30 elements in the composed signature, the error is of 0.0500 .

The results of these preliminary experiments with the modified tourist walk transient time and cycle period joint distribution with several memory values combined with a signature vector and a powerfull statistical multivariate analysis show the potential use of them as an efficient image classification method.

The new treatment proposed here diminishes the individual importance for each $\mu$ value, but stresses the role of small values either for $\mu$ or the transient time and cycle period. This conclusion is corroboreted with the small error taxes for the second experiment

\section{Conclusion}

We have presented a new method of feature extraction of image textures based on the deterministic tourist walk. The methods most commonly used deals with 
defined scales of pixel distributions. The distribution of transient times and periods of a set of data (image) present a wide range, capturing details on the organization of pixels from the micro to the macro scales and the resulting curve is strictly related to the configuration of the data set. We have showed that the joint distribution of the modified TW is an efficient tool for texture classification. We have realized two experiments using the modified TW and discriminant analysis to classify Brodatz textures. The results presented in this paper, show the great potential of the modified TW to be used as a texture analysis methodology.

\section{Acknowledgements}

O.M.B. acknowledges support from CNPq (303746/2004-1). M.G.C. acknowledges support from CAPES. A.S.M. acknowledges support from CNPq (305527/ 2004-5) and FAPESP (2005/02408-0 ).

\section{References}

1. Wu, C.M., Chen, Y.C., Hsieh, K.S.: Texture features for classification of ultrasonic liver images. IEEE Transactions on Medical Imaging 11 (1992) 141-152

2. Yang, C.Z.X.: Study of remote sensing image texture analysis and classification using wavelet. International Journal of Remote Sensing 19(16) (1998) 3197-3203

3. Heidelbach, F., Kunze, K., Wenk, H.R.: Texture analysis of a recrystallized quartzite using electron diffraction in the scanning electron microscope. Journal of Structural Geology (2000) 91-104

4. Anguiano, E., Oliva, A.I., Aguilar, M.: Surface texture parameters as a tool to measure image quality in scanning probe microscope. Ultramicroscopy $\mathbf{7 7}(3)$ (1999) $195-205$

5. Li, S.: On discontinuity-adaptive smoothness priors in computer vision. IEEE Transactions on Pattern Analysis and Machine Intelligence 17(6) (1995) 576-586

6. Howarth, P., Ruger, S.: Evaluation of texture features for content-based image retrieval. Lecture Notes in Computer Science 3115 (2004) 326-334

7. Jain, A.K., Farrokhnia, F.: Unsupervised texture segmentation using gabor filters. Pattern Recognition 24(12) (1991) 1167-1186

8. Campiteli, M.G., Martinez, A., Bruno, O.M.: A statisitcal texture classification framework using deterministic tourist walks - submited. INFOCOMP Journal of Computer Science (2006)

9. Campiteli, M.G., Martinez, A.S., Bruno, O.M.: An image analysis methodology based on deterministic walks - to appear in lecture notes in computer science. Ibero-American Conference on Artificial Intelligence (2006)

10. Scholl, J., Schol-Paschinger, E.: Classification by restricted random walks. Pattern Recognition 36 (2003) 1279-1290

11. Derrida, B.: From random walks to spin glasses. Phys. D 107(2-4) (1997) 186-198

12. Freund, H., Grassberger, P.: The Red Queens walk. Physica A 190(3-4) (1992) $218-237$

13. Bunimovich, L.A., Troubetzkoy, S.E.: Recurrence properties of Lorentz lattice gas cellular automata. J. Stat. Phys. 67(1-2) (1992) 289-302 
14. Bunimovich, L.A.: Deterministic walks in random environments. Physica D $\mathbf{1 8 7}$ (2004) 20-29

15. Gale, D., Propp, J., Sutherland, S., Troubetzkoy, S.: Further travels with my ant. Math. Inteligencer 17(3) (1995) 48-56

16. Lima, G.F., Martinez, A.S., Kinouchi, O.: Deterministic walks in random media. Phys. Rev. Lett. 87(1) (2001) 010603

17. Stanley, H.E., Buldyrev, S.V.: Statistical physics - the salesman and the tourist. Nature (London) 413(6854) (2001) 373-374

18. Kinouchi, O., Martinez, A.S., Lima, G.F., Loureno, G.M., Risau-Gusman, S.: Deterministic walks in random networks: an application to thesaurus graphs. Physica A 315(3/4) (2002) 665-676

19. Boyer, D., Miramontes, O., Ramos-Fernandez, G., Mateos, J.L., Cocho, G.: Modeling the searching behavior of social monkeys. Physica A 342(1-2) (2004) 329-335

20. Boyer, D., Larralde, H.: Looking for the right thing at the right place: Phase transition in an agent model with heterogeneous spatial resources. Complexity 10(3) (2005) 52-55

21. aes Campiteli, M.G., Batista, P.D., Kinouchi, O., Martinez, A.S.: Deterministic walks as an algorithm of pattern recognition. submitted. (2006)

22. Terçariol, C.A.S., Martinez, A.S.: Analytical results for the statistical distribution related to memoryless deterministic tourist walk: Dimensionality effect and mean field models. Phys. Rev. E 72 (2005) 021103

23. Brodatz, P.: A Photographic Album for Arts and Design. Volume 1. Dover Publishing Co., Toronto (1966)

24. R Development Core Team: R: A Language and Environment for Statistical Computing. R Foundation for Statistical Computing, Vienna, Austria. (2005)

25. Webb, A.: Statistical Pattern Recognition. Volume 1. Newnes (1999) 